\title{
An approximate analytic method for calculating the temperature field for a phase transition in an embankment in a permafrost region
}

\author{
Nansheng Li • Bo Tang
}

Received: 10 April 2014 / Accepted: 16 December 2014 / Published online: 31 December 2014

(C) The Author(s) 2014. This article is published with open access at Springerlink.com

\begin{abstract}
In this study, we applied conformal mapping and irregular perturbation methods to develop an analytic method to calculate the temperature field for a phase transition in the irregular domain of an embankment in a permafrost region. We then used this method to derive an approximate formula for the unstable temperature field of the phase transition in a homogeneous embankment over an irregular domain. First, we used conformal mapping to project a two-dimensional homogeneous embankment with irregular boundaries onto a one-dimensional semi-infinite area to derive the thermal conduction equations for unstable phase transitions and a continuity equation for the interfacial heat flux in the mapping coordinates. The irregularity of the boundary of the solution domain meant that only the mapping in the spatial domain could be used to inexpensively impose the boundary conditions analytically onto the regular boundary, thereby enabling the formulation of an analytical solution for the temperature field for a phase transition over an irregular domain. A small parameter perturbation and the orthogonal polynomial approximation were then used to analytically compute the thermal conduction equation for a phase transition in the mapping coordinate system.
\end{abstract}

\section{Introduction}

The Tibetan Plateau in China is the largest (in terms of area) mid-low latitude permafrost region in the world, with an area of approximately $1.47 \times 10^{6} \mathrm{~km}^{2}$. The Qinghai-Tibet

\section{N. Li $(\bowtie) \cdot$ B. Tang}

Department of Hydraulic Engineering, College of Civil Engineering, Tongji University, Shanghai 200092, China e-mail: linansheng@tongji.edu.cn railway and the Qinghai-Tibet highway, which are constructed on the Tibetan Plateau, are the longest permafrost railway and highway at the highest elevations in the world. The alternating transitions between positive and negative temperatures in permafrost areas during the winter and summer seasons induces a freeze-thaw cycle in the soil of the active layers of the railway and highway embankments, which damages the roadbed in the permafrost areas. The structural stability of the embankment is directly related to the change in the embankment temperature, especially during the phase transition of the pore water in the embankment soil (i.e., to the homogeneity of moisture migration through the embankment).

Calculating the temperature of a road embankment in permafrost areas involves solving a non-linear thermal conduction problem with a phase transition, which is a strongly non-linear computational problem with freemoving boundaries. The primary difficulties encountered in solving such problems are as follows: the non-linearity introduced by the discontinuous heat flux over the phase transition interface, the computational difficulties associated with the non-stationary phase interface, irregular boundaries, and non-uniform material properties. The vast majority of these problems do not have exact analytical solutions, and numerical and approximate analytical solutions are the only available options. For a thermal conduction problem with a phase transition, which involves nonlinear physical equations and irregular geometric shapes, we can apply an analytical perturbation method to derive an approximate formula for the temperature, as Caldwell and Kwan have shown [1]. However, non-linear problems with complicated geometric shapes are extremely difficult to solve. There are two primary difficulties: first, the non-linearity of the partial differential equations makes them hard to solve, and second, the analytical boundary 
condition cannot be easily applied to boundaries with arbitrary shapes. A variety of solution options have been used to address the first difficulty in the literature. Recently, Myers and Mitchell [2] developed a combined integral method (CIM) to solve the Stefan problem: the one-dimensional Stefan problem was solved in the infinite domain by directly integrating the thermal balance equation over the phase interface. Goto and Suzuki [3] combined a latent heat model with a boundary integral equation to develop a semi-analytical method to solve problems of transient consolidation and heat conduction during melting. Căruntu and Bota [4] introduced an approximate analytical method called the squared remainder minimization method (SRMM), which uses an increment polynomial to approximate an n-th non-linear thermal conduction differential equation and the corresponding thermal boundary conditions. The perturbation method is an approximate method, which being conceptually clear and intuitive and inexpensive to apply has been widely used to solve non-linear thermal conduction problems with phase transitions. We review the relevant literature over recent years here. Yigit [5] used a linear perturbation method to analyze a twodimensional phase transition problem for liquid consolidation on a planar mold surface from the heat flux of cold sources. Aziz and Benzies [6] used regular perturbation theory to derive an approximate solution for thermal conduction problems of temperature-dependent materials. Villatoro et al. [7] used a perturbation analysis based on the Laplace transform, in which the thermal conductivity was the small perturbation parameter, to derive an approximate analytical solution for a one-dimensional thermal conduction problem between an inert gas and a porous medium. Sadat and Prax [8] used perturbation theory to develop an explicit and simple analytical method to solve the thermal conduction problem for a solid affected by periodic heat sources. This method produced fairly accurate approximate solutions, i.e., first- and second-order perturbation solutions, for low-frequency cases. Even earlier (i.e., pre1980 's), perturbation methods were used to solve thermal conduction problems [9-12] mostly for regularly shaped boundaries and relatively simple working conditions. Chung et al. [13] used conformal mapping to map regions with buried pipelines from the semi-infinite domain to a rectangular region and derived a singular Fredholm integral equation of the second kind to solve a linear stable thermal conduction problem for the soil near the buried pipelines.

A solution domain with a complicated irregular boundary poses substantial challenges in the solution of thermal conduction problems. The boundary conditions for boundaries with complicated shapes are not "analytical", i.e., the values on these types of boundaries cannot be analytically introduced into the equation at one time. Thus, the analytical solution is not as feasible as a numerical computation for exerting the boundary conditions. The analytical computation is carried out continuously over the whole region, and the boundary conditions can only be easily applied to regular boundaries, whereas the numerical calculation is carried out on limited discrete points, such that the boundary conditions can easily be applied from one point to the next. Regional regularization is one of the most effective ways of obtaining an analytical solution over a complex domain. In this method, an irregular boundary is mapped into a regular boundary with regular shapes through a mapping (transformation) method. Conformal mapping [14] is an effective and convenient approach for region conversion, which converts a problem over relatively complicated regions into a problem over simpler regions. Moreover, conformal mapping is rotationally invariant, which is critical for solving thermal conduction problems. This requirement is significant because the calculations in this paper are based on the fundamental assumption that the orthogonality between the heat flux and the isotherms in the coordinate systems does not change before and after mapping in the thermal conduction problem.

The main contribution of this paper is the development of a fundamental thermal conduction equation for a phase transition using a mapping coordinate system based on the transformation relationship between a differential operator vector and the physical quantities in the conformal mapping. We also implement an approximate analytical calculation for the temperature field of the phase transition in an embankment by using a perturbation in a small parameter to solve a thermal conduction equation with function coefficients in the mapping coordinates. The asymptotic approximation is based on orthogonal basis functions, and the derived formulas are inversely applied to the original configuration of the embankment. To preserve the angles in the conformal mapping and the orthogonality between the isotherms and heat flux lines, we assume in the derivation that the front of the phase transition is slow in the mapping coordinates (i.e., the difference between the isothermal surface of the phase transition temperature and the horizontal surface is small) to simplify the calculation. Although we only use the lowest order term in the perturbation parameter in this paper, the expansion of the approximate perturbation converges very fast because the order of magnitude of the selected perturbation parameter is very small, resulting in a very highly accurate asymptotic approximate solution.

\section{Computational model for the temperature field of a phase transition in an embankment}

Road construction in permafrost areas is often based on the principle of permafrost protection, and the roadbed is 


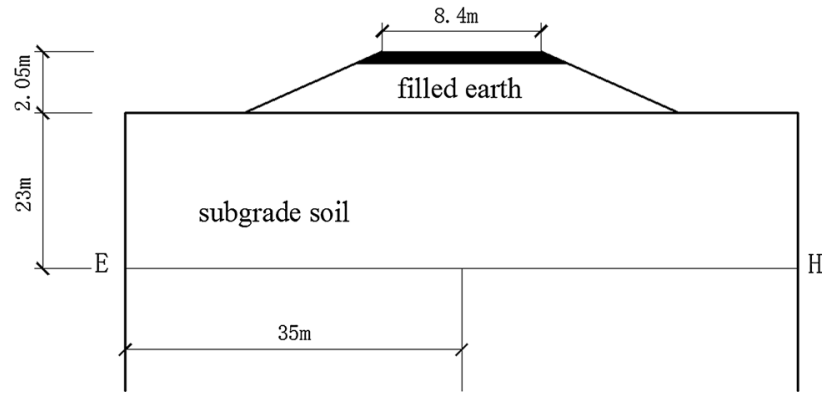

Fig. 1 Schematic of embankment cross section

usually filled. The roadbed cross section is trapezoidal, and the embankment can be approximated as layers of uniformly distributed filling material [15]; the embankment cross section is shown in Fig. 1.

The temperature field in the embankment in a permafrost region is controlled by the heat exchange between the land surface and atmosphere and between the underlying soil layer and the interior of the stratum. The climatic environment determines the depths of the maximum active layer and the stable temperature layer in the embankment. In addition, the embankment has a limited effect on the internal temperature field of the soil layer; thus, in calculating the embankment temperature field, the lower boundary $E H$ is modeled as a horizontal plane below a certain depth, and the temperature at the boundary is considered to be constant at $T_{G}$. To obtain analytical solutions, the roadbed discussed in this paper is oriented in the southnorth direction, and the shadier northern and sunny southfacing slope effect is neglected, along with the temperature differences between the side slope, the shoulder of the embankment and the pavement surface. The variation in the surface temperature is modeled by a cosine function in time. The layered structure of the material in the embankment cross section is neglected for the same reason, i.e., the embankment is considered to consist of homogeneous materials.

In this paper, we use a partial differential equation to model the isothermal phase transition temperature along with a front tracking method for the phase transition [16, 17]. Therefore, the two-dimensional unstable thermal conduction problem for the ice-water phase transition in the soil is formulated as follows:

$C_{j} \frac{\partial T_{j}}{\partial t}=\nabla \cdot\left(k_{j} \nabla T_{j}\right), \quad j=s, l$

$k_{j}= \begin{cases}k_{s} & T>T_{f} \\ k_{l} & T \leq T_{f}\end{cases}$

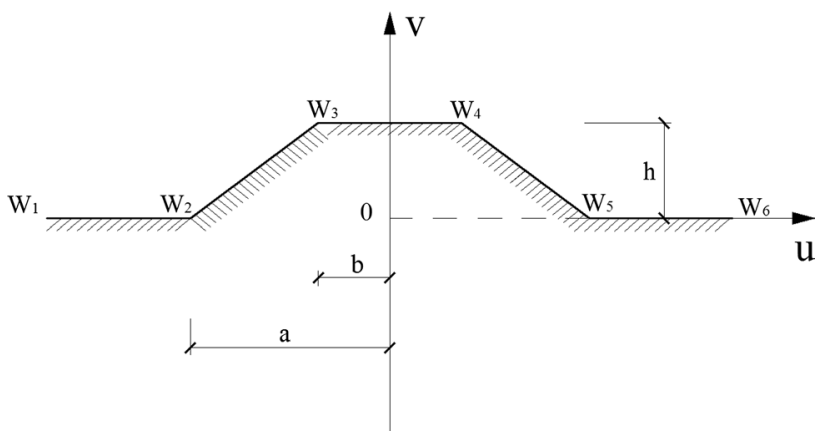

Fig. 2 Cross section of embankment and coordinate system before mapping

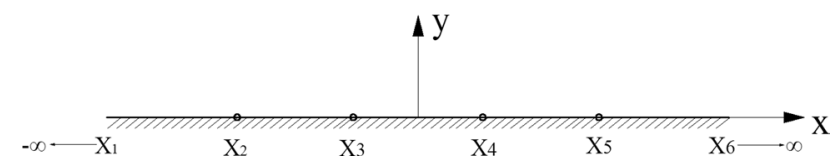

Fig. 3 Mapping coordinate system and semi-infinite domain

$C_{j}= \begin{cases}C_{s} & T>T_{f} \\ C_{l} & T \leq T_{f}\end{cases}$

$(\Delta \boldsymbol{q})_{n}=\rho L \frac{\partial S}{\partial t}$

The notation and units of the physical quantities in the aforementioned and following equations are given in "Appendix 1 ".

\section{Conformal mapping}

The boundary of the temperature field of the phase transition in the road embankment is an irregularly shaped polygon. An analytical solution cannot be formulated and solved using a unified coordinate system; thus, the boundary must be regularized before solving the problem with analytical methods. We used the Schwarz-Christoffel integral in the conformal mapping process $[14,18]$ process, i.e., we used the integral transform

$w=f(z)=C \int_{z_{0}}^{z} \prod_{k=1}^{n}\left(z-a_{k}\right)^{\alpha_{k}-1} d z+C_{1}$

to transform the polygonal region in $(u, v)$ coordinates to the semi-infinite domain in the mapping coordinates $(x, y)$.

Figure 2 shows the embankment cross section, Fig. 3 the semi-infinite system after mapping. The mapping relations of the coordinates are shown in Figs. 2 and 3. 
The conformal mapping function (which is derived in “Appendix 2") is

$$
\begin{aligned}
w= & (1.000005+18.854 \mathrm{i})[2.7071 z-0.1412(0.32465 \\
& \left.+\ln |\sqrt{2}+z|-\frac{1}{2} \ln \left|z^{2}-2\right|\right)+0.5074 \frac{z}{z^{2}-2} \\
& \left.-0.3382 \frac{z}{\left(z^{2}-2\right)^{2}}+0.9947 \frac{z}{\left(z^{2}-2\right)^{3}}\right] \\
& -(0.04584-\mathrm{i} h)
\end{aligned}
$$

The original Cartesian coordinate system $(u, v)$ is mapped to a new orthogonal coordinate system $(x, y)$ through the conformal mapping relationship in Eq. (3.2), which is used to derive the relationship between the generalized differential operators and the Jacobi determinant in orthogonal coordinates.

\section{Thermal conduction equation for a phase transition in mapping coordinates}

The conformal mapping function is the univalent analytic function

$w=f(z)=u(x, y)+\mathrm{i} v(x, y) z=x+\mathrm{i} y$

which represents a coordinate transformation from the $(u$, $v)$ plane to the $(x, y)$ plane. Therefore, both the thermal conduction equation and the spatial measurement of the physical quantities vary with the spatial coordinates in the new coordinate plane. In the plane of the mapping coordinates, Fourier's law in the direction of the mapping coordinate axis can be written as follows [19]:

$q_{m}=-\frac{k_{j}}{h_{m}} \nabla T$

where $h_{m}$ is the metric coefficient of the $m$ th mapping coordinate [20] (The functional form of the metric coefficient is derived in "Appendix 3").

The coordinate mapping deforms the space, thereby changing the material density. The relationship between the densities before and after the mapping is determined by the spatial mapping relationship. The unit cell $\mathrm{d} u \mathrm{~d} v$ in $(u, v)$ coordinates before the mapping corresponds to the unit cell $\mathrm{d} x \mathrm{~d} y$ in the mapping $(x, y)$ coordinates after the conformal mapping. Mass conservation requires that the two unit cells have equal masses before and after the transformation, i.e.,

$\rho d u d v=\rho^{\prime} d x d y \rho^{\prime}=\rho|J(x, y)|$

where $J(x, y)$ is the Jacobi matrix, an expression for which is given in "Appendix 3". The conformal mapping converts the thermal conduction problem in the original $(u, v)$ coordinates to a thermal conduction problem in the mapping $(x, y)$ coordinates, which changes the form of the thermal conduction equation. The conformal transformation relationship $w=f(z)=u(x, y)+i v(x, y)$ is used to derive the thermal conduction equation and the interface conditions in $(x, y)$ coordinates [21-23]:

$\frac{\partial T_{j}}{\partial t}=\alpha_{j}\left\{\frac{1}{h_{x}} \frac{\partial}{\partial x}\left[\frac{1}{h_{x}} \frac{\partial T_{j}(x, y ; t)}{\partial x}\right]+\frac{1}{h_{y}} \frac{\partial}{\partial y}\left[\frac{1}{h_{y}} \frac{\partial T_{j}(x, y ; t)}{\partial y}\right]\right\}$

$\frac{1}{h_{n}}\left[k_{s} \frac{\partial T_{s}(x, y ; t)}{\partial n}-k_{l} \frac{\partial T_{l}(x, y ; t)}{\partial n}\right]=\rho L|J(x, y)| \frac{\partial s(x ; t)}{\partial t}$

The metric coefficient $h_{x(y)}$ appears in Eq. (4.4). The metric coefficient $h_{n}$ appears in the left-hand side of the interface Eq. (4.5), and the coefficient on the right-hand side of the equation is the function $|J(x, y)|$. Although the thermal conduction equation has a more complex form in the mapping coordinates than in the original coordinates, the conformal mapping enables us to regularize the boundary such that the analytical boundary conditions can be applied, and the thermal conduction equation can be solved analytically.

After the mapping, the system satisfies the following initial, boundary, and interface conditions:

$T_{l}(x, y ; t=0)=T_{G}$

$T_{s}(x, 0 ; t)=T_{f}+\left(T_{f}-T_{G}\right) \operatorname{Re}\{A \exp [\mathrm{i}(\omega t+\varphi)]\}$

$T_{l}(x, y=\bar{X} ; t)=T_{G}$

$T_{s}=T_{l}=T_{f} \quad$ on $s(x ; t)$

In Eq. (4.7), $R e$ denotes taking the real part of a complex number. In the mapping coordinate plane $(x, y)$ (see Fig. 3), the initial and lower boundary $(y=\bar{X})$ temperatures for the embankment and the roadbed are set to the local multi-year average temperature $T_{G}$. In the permafrost region, the lower boundary temperature $T_{G}$ is lower than the melting temperature $T_{f}$ of the frozen soil.

\section{An approximate analytical solution for the unstable temperature field of the phase transition}

The conformal mapping transforms the solution domain for the irregular embankment to a one-dimensional semi-infinite space, resulting in a non-linear partial differential equation with variable coefficients for the thermal conduction problem with a phase transition. Currently, there no accurate analytical solutions exist for such complex non-linear partial differential equations; thus, obtaining an asymptotic analytical solution using a perturbation parameter is 
an alternative option [11, 12, 24]. In perturbation analysis, we first non-dimensionalize the physical quantities in the partial differential equation and the boundary, initial, and interface conditions. Thus, we define the following dimensionless quantities:

$\xi=\frac{x}{\bar{X}} \quad \eta=\frac{y}{\bar{X}} \quad \tau=\frac{\alpha_{s} t}{\bar{X}^{2}} \quad \gamma=\frac{\alpha_{s}}{\alpha_{l}} \quad \kappa=\frac{k_{l}}{k_{s}}$

$\varsigma=\frac{\alpha_{s}}{\omega \bar{X}^{2}} \quad U(\xi, \eta ; \tau)=\frac{T_{s}-T_{f}}{T_{f}-T_{G}} \quad V(\xi, \eta ; \tau)=\kappa \frac{T_{l}-T_{f}}{T_{f}-T_{G}}$

$v$ Ste $=\frac{C_{S}\left(T_{f}-T_{G}\right)}{L} \quad \delta=\frac{\bar{X}-s}{\bar{X}}$

Substituting the equations above into the partial differential Eq. (4.4), the interface condition (4.5) and the boundary and initial conditions yields

$\frac{\partial U(\xi, \eta ; \tau)}{\partial \tau}=\frac{1}{h_{\xi}} \frac{\partial}{\partial \xi}\left[\frac{1}{h_{\xi}} \frac{\partial U(\xi, \eta ; \tau)}{\partial \xi}\right]+\frac{1}{h_{\eta}} \frac{\partial}{\partial \eta}\left[\frac{1}{h_{\eta}} \frac{\partial U(\xi, \eta ; \tau)}{\partial \eta}\right]$

$\gamma \frac{\partial V(\xi, \eta ; \tau)}{\partial \tau}=\frac{1}{h_{\xi}} \frac{\partial}{\partial \xi}\left[\frac{1}{h_{\xi}} \frac{\partial V(\xi, \eta ; \tau)}{\partial \xi}\right]+\frac{1}{h_{\eta}} \frac{\partial}{\partial \eta}\left[\frac{1}{h_{\eta}} \frac{\partial V(\xi, \eta ; \tau)}{\partial \eta}\right]$

$\frac{\nu \text { Ste }}{h_{n}}\left\{\frac{\partial U[\xi,(1-\delta) ; \tau]}{\partial n}-\frac{\partial V[\xi,(1-\delta) ; \tau]}{\partial n}\right\}=-|J| \frac{\partial \delta}{\partial \tau}$

$V(\xi, \eta ; \tau=0)=-\kappa$

$V(\xi, \eta=1 ; \tau)=-\kappa$

$U(\xi, \eta=0 ; \tau)=\operatorname{Re}\left\{A \exp \left[\mathrm{i}\left(\frac{\tau}{\zeta}+\varphi\right)\right]\right\}$

Note that $\frac{\partial}{\partial n}$ in Eq. (5.4) represents the derivative in the normal direction relative to the interface of the phase transition in the transformed coordinates. The interface and initial conditions in the temperature at the phase transition front $\delta(\xi ; \tau)$ are as follows:

$U(\xi, 1-\delta ; \tau)=V(\xi, 1-\delta ; \tau)=0$

$\delta\left(\xi ; \tau=\tau_{0}\right)=1$

Equation (5.9) shows that the phase transition starts to develop at time $\tau=\tau_{0}$ (i.e., when the upper boundary condition first becomes positive), moving from the embankment surface to the deep part of the permafrost, which is the horizontal plane for the initial phase transition front of $\eta=0$ in the mapping coordinates.

The upper boundary condition is a periodic cosine function in the time variable $\tau$, i.e., Eq. (5.7); thus, it is reasonable to assume that the soil and boundary temperatures changing with time variable $\tau$ has the same frequency, so formulating the temperature and interface conditions by the variable separation method in the following form:

$V(\xi, \eta ; \tau)=V^{\prime}(\xi, \eta)\left\{A \exp \left[\mathrm{i}\left(\frac{\tau}{\zeta}+\varphi^{\prime}\right)\right]\right\}$

$U(\xi, \eta ; \tau)=U^{\prime}(\xi, \eta)\left\{A \exp \left[\mathrm{i}\left(\frac{\tau}{\zeta}+\varphi^{\prime}\right)\right]\right\}$

$\delta(\xi ; \tau)=1+\delta^{\prime}(\xi)\left\{A \exp \left[\mathrm{i}\left(\frac{\tau}{\zeta}+\varphi^{\prime}\right)\right]\right\}$

In the equations above, initial phase angle $\varphi^{\prime}$ can be determined by respective initial conditions, or are integrated into the solving procedure of undetermined functions $V^{\prime}(\xi, \eta), U^{\prime}(\xi, \eta), \delta^{\prime}(\xi)$ and they can also be complex; thus, the real parts of these functions are used in the final solution. The equations above are substituted into Eqs. (5.2)-(5.4) to yield the following equations:

$\frac{\mathrm{i}}{\zeta} U^{\prime}(\xi, \eta)=\frac{1}{h_{\xi}} \frac{\partial}{\partial \xi}\left[\frac{1}{h_{\xi}} \frac{\partial U^{\prime}(\xi, \eta)}{\partial \xi}\right]+\frac{1}{h_{\eta}} \frac{\partial}{\partial \eta}\left[\frac{1}{h_{\eta}} \frac{\partial U^{\prime}(\xi, \eta)}{\partial \eta}\right]$

$$
\mathrm{i} \frac{\gamma}{\zeta} V^{\prime}(\xi, \eta)=\frac{1}{h_{\xi}} \frac{\partial}{\partial \xi}\left[\frac{1}{h_{\xi}} \frac{\partial V^{\prime}(\xi, \eta)}{\partial \xi}\right]+\frac{1}{h_{\eta}} \frac{\partial}{\partial \eta}\left[\frac{1}{h_{\eta}} \frac{\partial V^{\prime}(\xi, \eta)}{\partial \eta}\right]
$$

$\frac{\varepsilon}{h_{\eta}}\left\{\frac{\partial U^{\prime}\left[\xi,\left(1-\delta^{\prime}\right)\right]}{\partial \eta}-\frac{\partial V^{\prime}\left[\xi,\left(1-\delta^{\prime}\right)\right]}{\partial \eta}\right\}=-\mathrm{i}|J| \delta^{\prime}(\xi)$

where $\varepsilon=v$ Ste $* \varsigma$ is the perturbation parameter. In deriving the interface Eq. (5.15), we make the following approximation: the upper boundary $(\eta=0)$ and the lower boundary $(\eta=1)$ in the one-dimensional mapping coordinates are at (or close to) the horizontal isothermal surfaces at a given time $\tau$, whereas the heat flux line remains perpendicular to the isothermal surfaces in the mapping coordinates; therefore, we assume that the derivative in the calculation direction, $\frac{\partial}{\partial n}$, at the isothermal phase transition interface can be approximated by $\frac{\partial}{\partial \eta}$.

To solve for approximate solutions to the equation above, we used the small parameter perturbation method [25] to expand $U^{\prime}(\xi, \eta), V^{\prime}(\xi, \eta)$, and $\delta^{\prime}(\xi)$ in a power series in the parameter $\varepsilon$ :

$U^{\prime}(\xi, \eta ; \varepsilon)=\sum_{n=0}^{\infty} \varepsilon^{n} U_{n}(\xi, \eta)$

$V^{\prime}(\xi, \eta ; \varepsilon)=\sum_{n=0}^{\infty} \varepsilon^{n} V_{n}(\xi, \eta)$ 
$\delta^{\prime}(\xi ; \varepsilon)=\sum_{n=0}^{\infty} \varepsilon^{n} \delta_{n}(\xi)$

The perturbation coefficient $\varepsilon$ used in the equations above is $\ll 1$ for a natural soil medium. Thus, we can retain only the lower order terms in the expanding Eqs. (5.16)(5.18) to obtain an approximate solution to the problem.

We substitute Eqs. (5.16)-(5.18) into the interface condition Eq. (5.15) and then expand the unknown functions in a Taylor series. Terms with the same power of $\varepsilon$ are compared to derive a low order equation for the interface position function $\delta_{n}(\xi)$ :

$\delta_{0}(\xi)=0$

$\sum_{j=0}^{\infty} \frac{\left(-\delta_{0}\right)^{j}}{j !}\left[\frac{\partial^{j+1} U_{0}(\xi, 1)}{\partial \eta^{j+1}}-\frac{\partial^{j+1} V_{0}(\xi, 1)}{\partial \eta^{j+1}}\right]=-\mathrm{i}|J| \delta_{1}(\xi)$

The front solution given by Eq. (5.19) for a phase transition is a null solution, and the first-order solution to Eq. (5.20) is not null only for $j=0$, indicating that a regular perturbation method cannot produce a physically meaningful solution for a phase transition front. To overcome this difficulty, we use the following transformation for the active layers of the embankment $(0 \leq \eta \leq \delta)$ :

$X=\frac{\eta}{\sqrt{\varepsilon}} \quad \bar{\delta}=-\frac{\delta^{\prime}(\xi)}{\sqrt{\varepsilon}} \quad \bar{U}(\xi, X ; \varepsilon)=U^{\prime}(\xi, X)$

Substituting the equation above into Eqs. (5.13), (5.15) and the boundary conditions yields

$\frac{i}{\varsigma} \bar{U}(\xi, X)=\frac{1}{h_{\xi}} \frac{\partial}{\partial \xi}\left[\frac{1}{h_{\xi}} \frac{\partial \bar{U}(\xi, X)}{\partial \xi}\right]+\frac{1}{\varepsilon h_{\eta}} \frac{\partial}{\partial X}\left[\frac{1}{h_{\eta}} \frac{\partial \bar{U}(\xi, X)}{\partial X}\right]$

$\frac{\partial \bar{U}\left(\xi, \frac{1}{\sqrt{\varepsilon}}+\bar{\delta}\right)}{\partial X}-\frac{\partial V^{\prime}[\xi,(1+\sqrt{\varepsilon} \bar{\delta})]}{\partial X}=\mathrm{i} \bar{\delta} \cdot h J[\xi,(1+\sqrt{\varepsilon} \bar{\delta})]$

$V^{\prime}[\xi,(1+\sqrt{\varepsilon} \bar{\delta})]=0$

$\bar{U}\left(\xi, \frac{1}{\sqrt{\varepsilon}}+\bar{\delta}\right)=0$

$\bar{U}(\xi, 0)=1$

In Eq. (5.23), $h J(\xi, \eta)=h_{\eta}(\xi, \eta) \cdot|J(\xi, \eta)|$.

Expanding $\bar{U}(\xi, X), \bar{\delta}(\xi, X)$ in a power series in the perturbation parameter $\varepsilon$ yields

$\bar{U}(\xi, X ; \varepsilon)=\sum_{n=0}^{\infty} \varepsilon^{n} \bar{U}_{n}(\xi, X)$ $\bar{\delta}(\varepsilon)=\sum_{n=0}^{\infty} \varepsilon^{n} \bar{\delta}_{n}$

Equations (5.23), (5.24), and (5.25) are all implicit equations in $\varepsilon$, which can be written as explicit functions of $\varepsilon$ using a Taylor series expansion:

$\bar{U}\left(\xi, \frac{1}{\sqrt{\varepsilon}}+\bar{\delta}\right)=\sum_{j=0}^{\infty} \frac{\left(\frac{1}{\sqrt{\varepsilon}}+\bar{\delta}\right)^{j}}{j !} \frac{\partial^{j} \bar{U}(\xi, 0)}{\partial X^{j}}=0$

$V^{\prime}[\xi,(1+\sqrt{\varepsilon} \bar{\delta})]=\sum_{j=0}^{\infty} \frac{(\sqrt{\varepsilon} \bar{\delta})^{j}}{j !} \frac{\partial^{j} V^{\prime}(\xi, 1)}{\partial X^{j}}=0$

$$
\begin{aligned}
& \sum_{j=0}^{\infty} \frac{\left(\frac{1}{\sqrt{\varepsilon}}+\bar{\delta}\right)^{j}}{j !} \frac{\partial^{j+1} \bar{U}(\xi, 0)}{\partial X^{j+1}}-\sum_{j=0}^{\infty} \frac{(\sqrt{\varepsilon} \bar{\delta})^{j}}{j !} \frac{\partial^{j+1} V^{\prime}(\xi, 1)}{\partial X^{j+1}} \\
& \quad=\mathrm{i} \bar{\delta} \sum_{j=0}^{\infty} \frac{(\sqrt{\varepsilon} \bar{\delta})^{j}}{j !} \frac{\partial^{j} h J(\xi, 1)}{\partial X^{j}}
\end{aligned}
$$

The perturbation solutions (5.27) and (5.28) are substituted into Eqs. (5.14), (5.22), (5.23) and the corresponding equations for the different conditions; the coefficients of the terms in the perturbation parameters $\left(\varepsilon^{-1}\right.$ and $\left.\varepsilon^{0}\right)$ of the same order on both sides of the equation are then equated to each other. The following equations are obtained for $\varepsilon^{-1}$ and $\varepsilon^{0}$ :

$\frac{\partial}{\partial X}\left[\frac{1}{h_{\eta}} \frac{\partial \bar{U}_{0}(\xi, X)}{\partial X}\right]=0$

$\mathrm{i} \frac{\gamma}{\zeta} V_{0}(\xi, \eta)=\frac{1}{h_{\xi}} \frac{\partial}{\partial \xi}\left[\frac{1}{h_{\xi}} \frac{\partial V_{0}(\xi, \eta)}{\partial \xi}\right]+\frac{1}{h_{\eta}} \frac{\partial}{\partial \eta}\left[\frac{1}{h_{\eta}} \frac{\partial V_{0}(\xi, \eta)}{\partial \eta}\right]$

$\sum_{j=0}^{\infty} \frac{\left(\bar{\delta}_{0}\right)^{j}}{j !} \frac{\partial^{j+1} \bar{U}_{0}(\xi, 0)}{\partial X^{j+1}}-\frac{\partial V_{0}(\xi, 1)}{\partial X}=\mathrm{i} h J(\xi, 1) \bar{\delta}_{0}$

$\bar{U}_{0}(\xi, 0)=1$

$\frac{\partial \bar{U}_{0}(\xi, 0)}{\partial X}$
$\quad=\left[1-\sum_{j=2}^{\infty} \frac{\left(-\bar{\delta}_{0}\right)^{j}}{j !} \frac{\partial^{j} \bar{U}_{0}(\xi, 0)}{\partial X^{j}}\right] /\left(-\bar{\delta}_{0}\right)\left(\right.$ or $\left.\bar{U}_{0}\left(\xi, \bar{\delta}_{0}\right)=0\right)$

Equation (5.32) is then combined with the boundary conditions in Eqs. (5.35) and (5.36) and $\frac{\partial h_{\eta}(\xi, X)}{\partial X} \rightarrow 0$. 
Setting the order of magnitude for the perturbation parameter as $\varepsilon \sim 10^{-6}$ yields

$\bar{U}_{0}(\xi, X)=\left\{\left[1-\sum_{j=2}^{\infty} \frac{\left(-\bar{\delta}_{0}\right)^{j}}{j !} \frac{\partial^{j} \bar{U}_{0}(\xi, 0)}{\partial X^{j}}\right] /\left(-\bar{\delta}_{0}\right)\right\} X+1$

Equation (5.37) can be used to obtain

$\frac{\partial^{j} \bar{U}_{0}(\xi, X)}{\partial X^{j}}=0 \quad j \geq 2$

Therefore,

$\bar{U}_{0}(\xi, X)=1-\frac{X}{\bar{\delta}_{0}}$

After various variable transformations, the function given above represents the equation for the temperature of the thawed soil phase. Equation (5.33) for the temperature of the homogenous frozen phase can then be combined with the following boundary conditions:

$V_{0}(\xi, 1)=-\kappa$

$V_{0}\left(\xi, 1-\delta_{0}\right)=0$

to describe heat conduction in the frozen phase.

The coefficients of the governing equation for the temperature field of the frozen phase are transcendental functions and thus cannot be solved using conventional mathematical and physics methods. Thus, we used the Galerkin method, which is based on a variational principle [26], to solve the equation. Trial solutions of orthogonal functions that satisfy the corresponding problem boundary conditions form the basis of an approximate solution to the partial differential equation with function coefficients [27, 28]. This approximate solution satisfies the orthogonality conditions of the complete basis functions, which are used to derive the undetermined coefficients of the asymptotic solution in the form of an orthogonal polynomial expansion, which in turn can be substituted into the asymptotic solution to derive an approximate solution to the partial differential equation with function coefficients.

A trial solution for the non-homogeneous partial differential equation for thermal conduction in the frozen soil can be approximated by an orthogonal polynomial, $V_{0}(\xi, \eta)$ :

$V_{0}(\xi, \eta)=-\left(\eta+\delta_{0}-1\right) \frac{\kappa}{\delta_{0}}+\sum_{l=0}^{N} \sum_{k=0}^{M} A_{k l} \varphi_{k}(\eta) \psi_{l}(\xi)$

where the independent variables $\eta \in\left[\delta_{0}, 1\right], \xi \in[-L, L]$, $\varphi_{k}(\eta)$, and $\psi_{l}(\xi)$ are Fourier polynomials (which are given in "Appendix 4").
Introducing the trial solution given by Eq. (5.42) into the governing Eq. (5.33) yields

$$
\begin{gathered}
\mathrm{i} \frac{\gamma}{\zeta}|J|\left\{-\left(\eta+\delta_{0}-1\right) \frac{\kappa}{\delta_{0}}+\sum_{l=0}^{N} \sum_{k=0}^{M} A_{k l} \varphi_{k}(\eta) \psi_{l}(\xi)\right\} \\
=\sum_{l=0}^{N} \sum_{k=0}^{M} A_{k l}\left[\varphi_{k}^{\prime \prime}(\eta) \psi_{l}(\xi)+\varphi_{k}(\eta) \psi_{l}^{\prime \prime}(\xi)\right]
\end{gathered}
$$

Next, we introduce the complete eigenfunctions for the orthogonal basis functions (see "Appendix 4"):

$\psi_{l}^{\prime \prime}(\xi)+\alpha_{l} \psi_{l}(\xi)=0$

$\varphi_{k}^{\prime \prime}(\eta)+\beta_{k} \varphi_{k}(\eta)=0$

Substituting the two equations above into Eq. (5.43) yields

$$
\begin{gathered}
\mathrm{i} \frac{\gamma}{\zeta}|J|\left\{-\left(\eta+\delta_{0}-1\right) \frac{\kappa}{\delta_{0}}+\sum_{l=0}^{N} \sum_{k=0}^{M} A_{k l} \varphi_{k}(\eta) \psi_{l}(\xi)\right\} \\
=-\sum_{l=0}^{N} \sum_{k=0}^{M} A_{k l}\left(\alpha_{l}+\beta_{k}\right) \varphi_{k}(\eta) \psi_{l}(\xi)
\end{gathered}
$$

We then expand $\frac{\left(\eta+\delta_{0}-1\right)}{\delta_{0}}|J|$ in Eq. (5.46) in a series of the complete basis functions $\varphi_{k}(\eta)$ and $\psi_{l}(\xi)$ :

$\frac{\left(\eta+\delta_{0}-1\right)}{\delta_{0}}|J|=\sum_{l=0}^{N} \sum_{k=0}^{M} C_{k l} \varphi_{k}(\eta) \psi_{l}(\xi)$

where

$C_{k l}=\int_{\delta_{0}}^{1} \int_{-L}^{L} \frac{\left(\eta+\delta_{0}-1\right)}{\delta_{0}}|J| \bar{\varphi}_{k}(\eta) \bar{\psi}_{l}(\xi) \mathrm{d} \eta \mathrm{d} \xi$

and $\bar{\varphi}_{k}(\eta)$ and $\bar{\psi}_{l}(\xi)$ are the conjugate Fourier basis functions. The second term on the left-hand side of Eq. (5.43) can be expanded as follows:

$|J| \sum_{l=0}^{N} \sum_{k=0}^{M} A_{k l} \varphi_{k}(\eta) \psi_{l}(\xi)=\sum_{s=0}^{M} \sum_{t=0}^{N} B_{s t} \varphi_{s}(\eta) \psi_{t}(\xi)$

where

$B_{s t}=\sum_{l=0}^{N} \sum_{k=0}^{M} \int_{\delta_{0}}^{1} \int_{-L}^{L}|J| A_{k l} \varphi_{k}(\eta) \psi_{l}(\xi) \bar{\varphi}_{s}(\eta) \bar{\psi}_{t}(\xi) \mathrm{d} \eta \mathrm{d} \xi$

Let us define the following term:

$D_{k l, s t}=\int_{\delta_{0}}^{1} \int_{-L}^{L}|J| \varphi_{k}(\eta) \psi_{l}(\xi) \bar{\varphi}_{s}(\eta) \bar{\psi}_{t}(\xi) \mathrm{d} \eta \mathrm{d} \xi$ 
Table 1 Thermal parameters for a homogenous embankment

\begin{tabular}{lllllll}
\hline Soil material & $\begin{array}{l}\text { Bulk density, } \\
\rho\left(\mathrm{kg} \mathrm{m}^{-3}\right)\end{array}$ & $\begin{array}{l}\text { Thermal conductivity, } \\
k\left(\mathrm{~W} \mathrm{~m}^{-1}{ }^{\circ} \mathrm{C}^{-1}\right)\end{array}$ & & $\begin{array}{l}\text { Specific heat capacity, } \\
C\left(\mathrm{~J} \mathrm{~kg}^{-1}{ }^{\circ} \mathrm{C}^{-1}\right)\end{array}$ & $\begin{array}{l}\text { Latent heat of phase } \\
\text { transition, } L\left(\mathrm{~J} \mathrm{~kg}{ }^{-1}\right)\end{array}$ \\
\cline { 2 - 5 } & Frozen soil & Melted soil & & Frozen soil & Melted soil \\
\hline Clayey (mild clay) & 1,600 & 2.12 & 1.42 & 1,222 & 1,608 & $3.769 \times 10^{4}$ \\
\hline
\end{tabular}

Substituting Eqs. (5.48), (5.50), and (5.51) into Eq. (5.46) yields

$$
\begin{gathered}
\left\{\sum_{l=0}^{N} \sum_{k=0}^{M}-\mathrm{i} \frac{\gamma \kappa}{\varsigma} C_{k l} \varphi_{k}(\eta) \psi_{l}(\xi)+\sum_{l=0}^{N} \sum_{k=0}^{M} \mathrm{i} \frac{\gamma}{\varsigma} B_{k l} \varphi_{k}(\eta) \psi_{l}(\xi)\right\} \\
=-\sum_{l=0}^{N} \sum_{k=0}^{M} A_{k l}\left(\alpha_{l}+\beta_{k}\right) \varphi_{k}(\eta) \psi_{l}(\xi)
\end{gathered}
$$

Comparing the coefficients of the same terms on both sides of the equation yields

$-\mathrm{i} \frac{\gamma \kappa}{\varsigma} C_{k l}+\mathrm{i} \frac{\gamma}{\varsigma} B_{k l}=-A_{k l}\left(\alpha_{l}+\beta_{k}\right)$

Let us define the following matrices:

$\boldsymbol{A}=\left(A_{k l}\right) \boldsymbol{D}=\left(D_{s t}, k l\right) \boldsymbol{C}=\left(C_{k l}\right) \Lambda=\operatorname{diag}\left(\alpha_{l}+\beta_{k}\right)$

Equation (5.53) can then be written in matrix form as follows:

$-\mathrm{i} \frac{\gamma \kappa}{\zeta} \boldsymbol{C}+\mathrm{i} \frac{\gamma}{\zeta} \boldsymbol{D A}=-\boldsymbol{\Lambda} \boldsymbol{A}$

The equation above can then be solved for $\mathbf{A}$ as follows:

$\boldsymbol{A}=\mathrm{i} \frac{\gamma \kappa}{\varsigma}\left(\mathrm{i} \frac{\gamma}{\zeta} \boldsymbol{D}+\boldsymbol{\Lambda}\right)^{-1} \boldsymbol{C}$

Substituting Eqs. (5.39) and (5.42) into the phase transition interface condition (5.34) and using Eq. (5.38) shows that the first term in Eq. (5.34), $\sum_{j=0}^{\infty} \frac{\left(\bar{\delta}_{0}\right)^{j}}{j !} \frac{\partial^{j+1} \bar{U}_{0}(\xi, 0)}{\partial X^{j+1}}$, only needs to be expanded up to the first term. The following equation is thus obtained for the phase transition interface:

$$
\begin{aligned}
-\frac{1}{\bar{\delta}_{0}}+ & \frac{\sqrt{\varepsilon} \kappa}{\delta_{0}}-\sqrt{\varepsilon}\left(\eta+\delta_{0}-1\right) \sum_{l=0}^{N} \sum_{k=0}^{M} A_{k l} \varphi_{k}^{\prime}(1) \psi_{l}(\xi) \\
= & \mathrm{i} h J(\xi, 1) \bar{\delta}_{0}
\end{aligned}
$$

The parameter for the phase transition interface, $\bar{\delta}_{0}$, appears on both sides of Eq. (5.57), making it somewhat cumbersome to derive an accurate solution to this equation. However, the terms with coefficients of $\sqrt{\varepsilon}$ can be neglected because the perturbation parameter $\varepsilon$ in
Eq. (5.57) is small. The transformation given by Eq. (5.21) is used to obtain an expression for $\bar{\delta}_{0}$ :

$\bar{\delta}_{0}=\sqrt{\frac{\mathrm{i}(1+\kappa)}{h J(\xi, 1)}}$

The real part of the solution to Eq. (5.58) is the zerothorder term of the phase transition front.

\section{Example calculations}

To verify the accuracy of the approximate method developed in this study, some of the approximate analytical results are compared with computational results from finite element analysis. The example calculations use the following geometric model of the embankment (the geometric configuration is shown in Fig. 1): a roadbed width of $8.4 \mathrm{~m}$, a roadbed height of $2.05 \mathrm{~m}$, a roadbed slope of $1: 1$; a calculated roadbed width of $70 \mathrm{~m}$, which extends from the center of roadbed to both sides for $35 \mathrm{~m}$; and a calculated depth of the roadbed that is $23 \mathrm{~m}$ below the original surface at the center of roadbed. The thermal parameters for the embankment soil are shown in Table 1.

The temperature at the upper boundary of embankment is given by

$T_{u}(t)=T_{f}+A \cos \left(6.342 \times 10^{-8} \pi t+\varphi\right)$

where $A=21.5^{\circ} \mathrm{C}$ and $\varphi=2.1981$, and the units of the time $\mathrm{t}$ are in second. The temperature at the lower boundary is $T_{G}=-6.75^{\circ} \mathrm{C}$, and the corresponding depth is $\bar{X}=23 \mathrm{~m}$. The initial temperature of the entire embankment, including the roadbed, is $T(x, y ; 0)=-6.75^{\circ} \mathrm{C}$, and the phase transition temperature of the soil is $T_{f}=-0.1^{\circ} \mathrm{C}$.

In the derivation process, the transformation relation and the inversion formation in the conformal mapping must be substituted into each set of equations derived above. The equation for the temperature field and the function for the phase transition front are inversely derived using the original coordinates $(u, v)$ of the embankment cross section as the independent variables. 


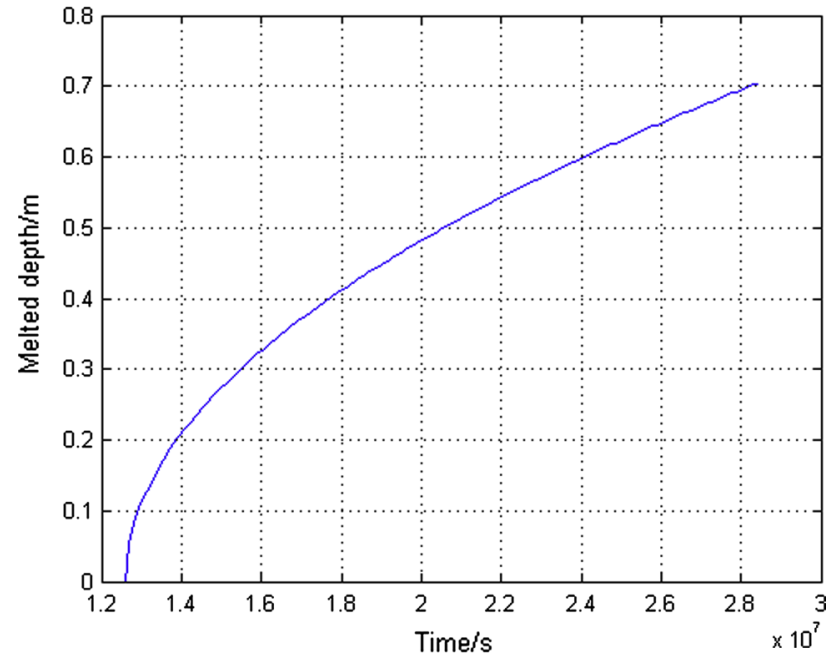

Fig. 4 Temporal curve of the phase transition front

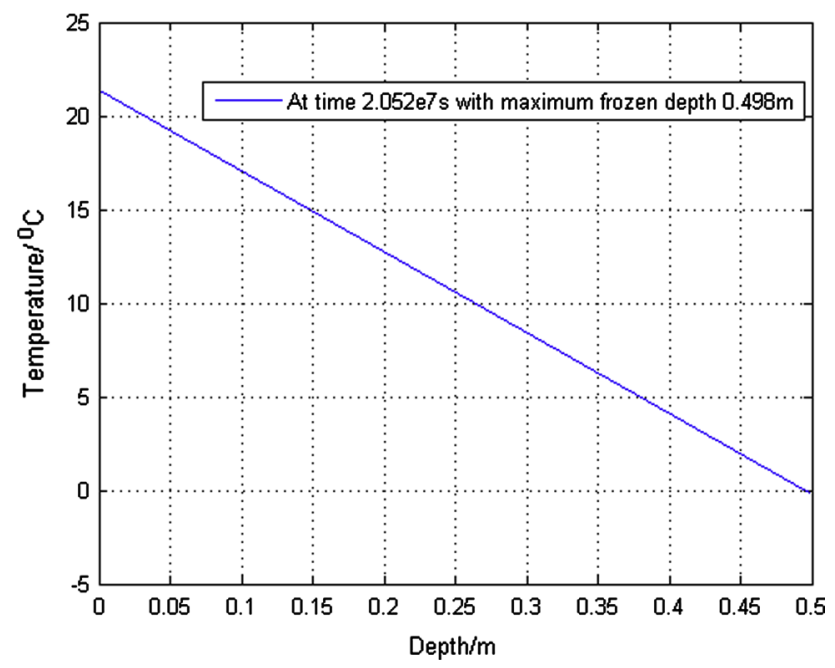

Fig. 5 Thawed soil temperature variation vs depth at $t=2.052 \times 10^{7} \mathrm{~s}$

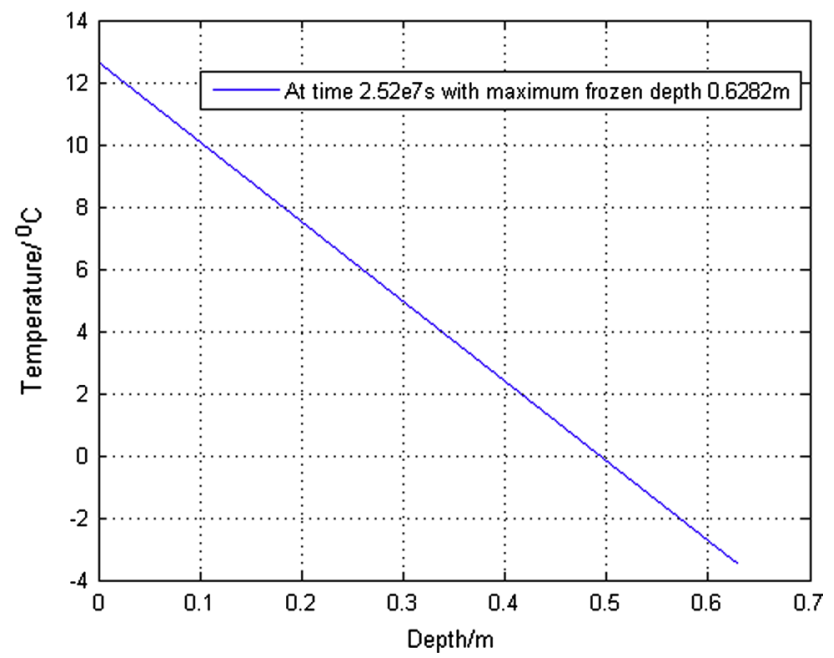

Fig. 6 Thawed soil temperature variation vs depth at $t=2.52 \times 10^{7} \mathrm{~s}$

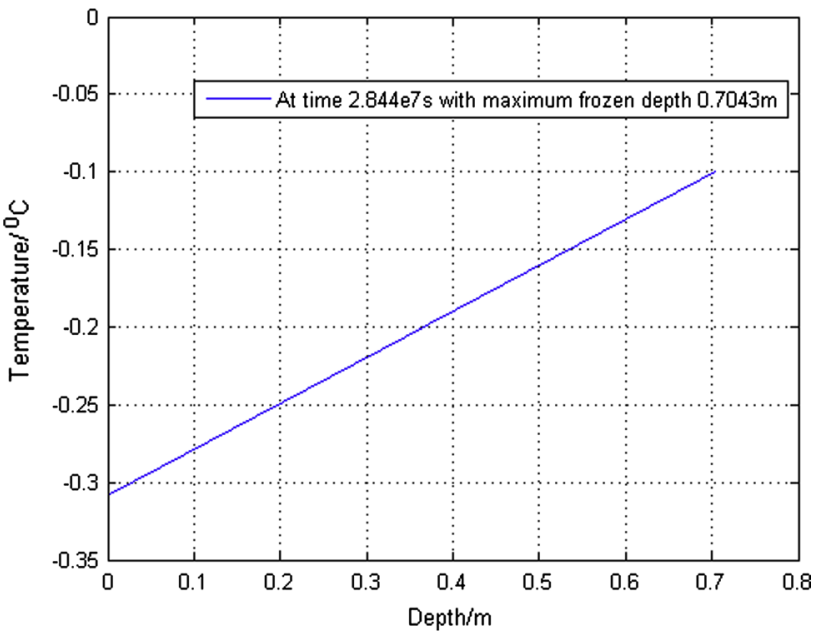

Fig. 7 Thawed soil temperature variation vs depth at $t=2.844 \times 10^{7} \mathrm{~s}$

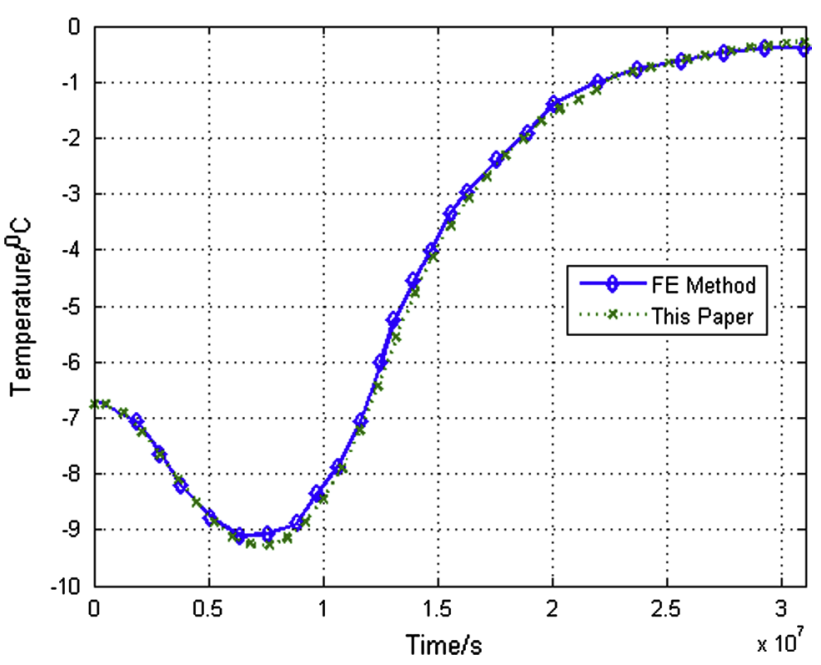

Fig. 8 Temperature change at the coordinates $[0,0]$

\subsection{Temporal evolution of the phase transition front}

For times $t>t_{0}$ (where $t_{0}=1.26 \times 10^{7} \mathrm{~s}$ is the time when the temperature at the upper boundary first becomes positive), the permafrost embankment starts to melts from the upper part, forming an active layer until refreezing occurs, i.e., when the temperature of the upper part of the embankment drops to the freezing temperature again (at time $t_{1}=2.844 \times 10^{7} \mathrm{~s}$ ). Equation (5.59) is substituted into Eq. (5.12) and the conversion Eqs. (5.21) and (5.1) are applied. We then used the conformal mapping relationship (3.2) to inversely derive the original coordinates $(u, v)$, yielding the temporal function of the phase transition front. Figure 4 shows the position profile of the phase transition front on the symmetric axis of embankment over the time interval $\left[t_{0}, t_{1}\right]$. 
6.2 Temperature change in the active embankment layers in permafrost areas

After a variety of variable transformations, the temperature of the thawing soil, i.e., Eq. (5.39), deceptively appears to be a simple first-order function in the x-coordinate. However, the solution equation contains the interface position $\bar{\delta}_{0}(\xi)$, and the physical variables undergo a series of transformations and a spatial mapping while solving for the solutions; thus, the temperature of the thawing soil is actually a complicated function of the spatial coordinates and time. In unidirectional melting, the thawing soil is located over the phase transition front, i.e., the vertical coordinate of the thawing soil, $v<s(u ; t)$. Figures 5, 6 and 7 show the temperature variation with depth in the direction of symmetric axis of embankment at times $t=2.052 \times 10^{7} \mathrm{~s}, 2.52 \times 10^{7} \mathrm{~s}$ and $2.844 \times 10^{7} \mathrm{~s}$. It worth noting from Fig. 7 that it's the time at $t=2.844 \times 10^{7} \mathrm{~s}$ to refreeze on the symmetric axis of embankment.

\subsection{Temporal curve for fixed embankment positions}

There is no consensus on standard criteria to validate a nonlinear temperature field for phase transitions such as freezethaw cycles for an embankment in a permafrost region. In this paper, we verify the approximate analytical solution for the temperature by comparison with computation results from finite element analysis. To improve the computational accuracy of the finite element solution, we divide the geometric configuration of the embankment into fine finite element meshes, each of a $10^{-2} \mathrm{~m}$ side. Figure 8 compares the timeresolved temperature at the coordinates $[0,0]$, showing that the approximate analytical solution developed in this paper is in excellent agreement with the finite element solution.

\section{Conclusions}

Few accurate analytical solutions exist for non-linear thermal conduction problems over irregular domains, such as the temperature field for a phase transition in an embankment in a permafrost region. In the analytical calculation, the irregularity of the domain causes greater computational difficulties than the soil phase transition. The boundary of a complicated domain must be described using functions of coordinate variables. Unlike in numerical calculations, the boundary conditions for thermal conductivity problems with phase transitions cannot be applied from one point to the next over the domain boundary in an analytical calculation. Therefore, conformal mapping is used in this paper to regularize the irregular domain, such that the boundary of the phase transition thermal conduction problem is transformed to a regular boundary with constant coordinate values in the mapping coordinates. This approach enables us to formulate an analytical solution even though the partial differential equations for the temperature field in the frozen and melting areas are transformed from a linear equation to non-linear equations with function coefficients.

The inherent complexity of thermal conduction problems with phase transitions requires the use of assumptions and approximations to generate analytical solutions. The key assumptions used in this paper have a sound theoretical basis. That is, the directional derivative at the phase transition front is approximated by preserving the angles in the conformal mapping, and the analytical results are validated by comparison with computational results. The perturbation method consists of an approximate series expansion of the solution in the perturbation parameter; thus, the convergence and accuracy of the approximate solutions depends considerably on the magnitude of the perturbation parameters. In this paper, the magnitude of the adopted perturbation parameters is very small. Thus, very high numerical accuracy can be achieved by using only the lowest order terms in the expansion, thereby avoiding having to solve very complicated different equations with higher order terms.

Finally, we should to emphasize that developing an analytical solution to a thermal conduction problem with a phase transition for irregular boundaries (such as for an embankment in a permafrost region) remains highly challenging even when the material inhomogeneity in the embankment structure is neglected. Considerable work remains to be performed to develop an analytical solution for a non-linear thermal conduction problem with irregular boundaries. To the best of our knowledge, very few studies exist on this subject. The assumptions we have made to generate an approximate analytical solution are compromises between the tractability of the calculation scheme and the accuracy of the results to a complex problem. Here, we have obtained a considerably high accuracy in the analytical results by selecting an appropriate perturbation parameter.

Acknowledgments Project supported by the National Natural Science Foundation of China (Grant No. 51179129).

Open Access This article is distributed under the terms of the Creative Commons Attribution License which permits any use, distribution, and reproduction in any medium, provided the original author(s) and the source are credited.

\section{Appendix 1: Nomenclature}

i Complex number

Complex plane $z=x+\mathrm{i} y,(x, y)$ are the mapping coordinates

Complex plane $w=u(x, y)+\mathrm{i} v(x, y),(u, v)$ are the original coordinates 


$$
\begin{aligned}
& h_{m} \quad \text { Metric coefficient of the } m \text { th mapping } \\
& \text { coordinate } \\
& \nabla \quad \text { Hamilton operator } \\
& \rho \quad \text { Material density }\left(\mathrm{kg} \mathrm{m}^{-3}\right) \text { in the original } \\
& \text { coordinate system } \\
& \rho^{\prime} \quad \text { Material density }\left(\mathrm{kg} \mathrm{m}^{-3}\right) \text { in the mapping } \\
& \text { coordinate system } \\
& \Delta \boldsymbol{q} \quad \text { Increment in the heat flux } \\
& \boldsymbol{q}=k(\nabla T) \quad \text { Heat flux }\left(\mathrm{W} \mathrm{m}^{2}\right) \\
& J(x, y)=\left[\frac{\partial(u, v)}{\partial(x, y)}\right] \text { Jacobi matrix } \\
& |J(x, y)| \quad \text { Jacobi determinant } \\
& j=l, s \quad \text { Index corresponding to frozen and } \\
& \text { thawed soil phases, respectively } \\
& T_{s} \quad \text { Temperature of thawed soil phase }\left({ }^{\circ} \mathrm{C}\right) \\
& T_{l} \quad \text { Temperature of frozen soil phase }\left({ }^{\circ} \mathrm{C}\right) \\
& s(u) \quad \text { Function for phase transition front } \\
& t \quad \text { Time (h) } \\
& k \quad \text { Thermal conductivity }\left(\mathrm{W} \mathrm{m} \mathrm{m}^{-1}{ }^{\circ} \mathrm{C}^{-1}\right) \\
& L \quad \text { Latent heat of phase transition }\left(\mathrm{J} \mathrm{kg}^{-1}\right) \\
& w_{1}=-\infty, \quad w_{2}=-a, \quad w_{3}=-b+\mathrm{i} h, \\
& w_{4}=b+\mathrm{i} h, \quad w_{5}=a, \quad w_{6}=\infty \\
& \left|w_{3}-w_{2}\right|=l_{2}=\sqrt{(a-b)^{2}+h^{2}}, \quad\left|w_{4}-w_{3}\right|=2 b=l_{3}, \\
& \left|w_{5}-w_{4}\right|=l_{4}=\sqrt{(a-b)^{2}+h^{2}} \\
& \left|x_{4}-x_{3}\right|=\frac{l_{3}}{l_{2}} \therefore x_{4}=\frac{b}{\sqrt{(a-b)^{2}+h^{2}}} \\
& \left|x_{5}-x_{4}\right|=\frac{l_{4}}{l_{2}} \therefore x_{5}=1+\frac{b}{\sqrt{(a-b)^{2}+h^{2}}} \text {. }
\end{aligned}
$$$$
\alpha=\frac{k}{\rho C} \quad \text { Thermal diffusivity }\left(\mathrm{m}^{2} \mathrm{~h}^{-1}\right)
$$$$
\gamma=\frac{\alpha_{s}}{\alpha_{l}} \quad \text { Dimensionless constant }
$$$$
\kappa=\frac{k_{l}}{k_{s}} \quad \text { Dimensionless constant }
$$$$
C \quad \text { Thermal capacity }\left(\mathrm{J} \mathrm{kg}^{-1}{ }^{\circ} \mathrm{C}^{-1}\right)
$$$$
T_{G} \quad \text { Multi-year average ground temperature }
$$$$
\text { of permafrost }\left({ }^{\circ} \mathrm{C}\right)
$$$$
T_{f} \quad \text { Phase transition temperature for soil }\left({ }^{\circ} \mathrm{C}\right)
$$$$
\bar{X} \quad \text { Characteristic depth, i.e., the minimum }
$$$$
\text { depth (m) at which the embankment }
$$$$
\text { affects the permafrost temperature }
$$$$
(\xi, \eta) \quad \text { Dimensionless coordinates }
$$$$
\tau \quad \text { Dimensionless time }
$$$$
U(\xi, \eta ; \tau) \quad \text { Dimensionless temperature of thawing }
$$$$
\text { soil phase }
$$$$
V(\xi, \eta ; \tau) \quad \text { Dimensionless temperature of frozen }
$$$$
v \text { Ste }=\frac{C_{s}\left(T_{f}-T_{G}\right)}{L} \stackrel{\text { phase }}{\text { Pseudo Stefan number }}
$$$$
\omega \quad \text { Frequency of periodic temperature }
$$$$
\text { change of embankment surface }
$$$$
\varsigma=\frac{\alpha_{s}}{\omega \bar{X}^{2}}
$$$$
\varepsilon=\zeta \cdot v \text { Ste }
$$$$
\delta(\xi ; \tau)
$$$$
\text { Dimensionless parameter }
$$$$
\text { Perturbation coefficient }
$$$$
\text { Dimensionless function for the phase }
$$$$
\text { transition front }
$$

superscript "'” A Fourier-transformed physical variable $X=\frac{\eta-1}{\varepsilon} \quad$ Vertical coordinate in the embankment active layer $(0 \leq \eta \leq \delta)$

superscript “-” Physical variables in the embankment active layer

\section{Appendix 2: Derivation of conformal mapping relation}

Figures 2 and 3 show the embankment cross section and the semi-infinite system after the mapping.
This mapping relation maintains a good mapping symmetry.

The Schwarz-Christoffel mapping function is

$$
\begin{aligned}
w=f(z)= & k \int\left[\left(z-x_{2}\right)^{\frac{\alpha_{2}}{\pi}-1}\left(z-x_{3}\right)^{\frac{\alpha_{3}}{\pi}-1}\right. \\
& \left.\left(z-x_{4}\right)^{\frac{\alpha_{4}}{\pi}-1}\left(z-x_{5}\right)^{\frac{\alpha_{5}}{\pi}-1}\right] d z+c .
\end{aligned}
$$

Let $a-b=\mathrm{h}, b=2 \mathrm{~h}$, then $x_{2}=-1-\sqrt{2}, x_{3}=-\sqrt{2}$, $x_{4}=\sqrt{2}$, and $x_{5}=1+\sqrt{2}$.

$$
\begin{aligned}
& \alpha_{2}=\frac{5 \pi}{4}, \alpha_{3}=\frac{3 \pi}{4}, \alpha_{4}=\frac{3 \pi}{4}, \alpha_{5}=\frac{5 \pi}{4} \\
& w=k \int\left[(z+(1+\sqrt{2}))^{\frac{1}{4}}(z+\sqrt{2})^{-\frac{1}{4}}(z-(1+\sqrt{2}))^{\frac{1}{4}}\right] \\
& d z+c=k \int\left[1-\frac{1+2 \sqrt{2}}{z^{2}-2}\right]^{\frac{1}{4}} d z+c
\end{aligned}
$$

Let us define the following terms:

$$
\begin{aligned}
& \frac{1+2 \sqrt{2}}{z^{2}-2}=\xi, \quad z=\left(\frac{1+2 \sqrt{2}}{\xi}+2\right)^{\frac{1}{2}}, \\
& w=-\frac{1+2 \sqrt{2}}{2 \sqrt{2}} k \int(1-\xi)^{\frac{1}{4}} \cdot\left(\frac{1+2 \sqrt{2}}{2}+\xi\right)^{-\frac{1}{2}} \xi^{-\frac{3}{2}} d \xi
\end{aligned}
$$

Using the following binomial series,

$$
\begin{gathered}
(1-\xi)^{\frac{1}{4}}=1-\frac{1}{4} \xi-\frac{1 \cdot 3}{4 \cdot 8} \xi^{2}-\frac{1 \cdot 3 \cdot 7}{4 \cdot 8 \cdot 12} \xi^{3} \\
-\frac{1 \cdot 3 \cdot 7 \cdot 11}{4 \cdot 8 \cdot 12 \cdot 16} \xi^{4}-\cdots
\end{gathered}
$$


we can determine $k$ and $c$ as follows

$$
\begin{aligned}
k= & 0.7388+6.7947 h \mathrm{i} \quad c=-0.0458+\mathrm{i} h . \\
w= & 1.3536 k\left\{2.7071 z-0.1412\left[0.32465+\ln |\sqrt{2}+z|-\frac{1}{2} \ln \left|z^{2}-2\right|\right]\right. \\
& \left.+\frac{0.50742 z}{z^{2}-2}-\frac{0.338177 z}{\left(z^{2}-2\right)^{2}}+\frac{0.99472 z}{\left(z^{2}-2\right)^{3}}\right\}+c \\
= & (1.000005+18.854 \mathrm{i}) \\
& \times\left[2.7071 z-0.1412\left(0.32465+\ln |\sqrt{2}+z|-\frac{1}{2} \ln \left|z^{2}-2\right|\right)\right] \\
& +0.5074 \frac{z}{z^{2}-2}-0.3382 \frac{z}{\left(z^{2}-2\right)^{2}}+0.9947 \frac{z}{\left(z^{2}-2\right)^{3}} \\
& -(0.04584-\mathrm{i} h)
\end{aligned}
$$

\section{Appendix 3: Metric coefficient $h_{m}$ and Jacobi matrix $J(x, y)$}

The metric coefficient and Jacobi matrix $J(x, y)$ along the direction of mapping coordinates $(x, y)$ are derived as follows. The mapping function is given by

$w=f(z)=u(x, y)+i v(x, y)=k \int\left[1-\frac{1+2 \sqrt{2}}{z^{2}-2}\right]^{\frac{1}{4}} \mathrm{~d} z$.

Since $w$ is an analytic function, the following relationships hold:

$\frac{\partial u}{\partial x}=\frac{\partial v}{\partial y} \quad \frac{\partial u}{\partial y}=-\frac{\partial v}{\partial x}$

Therefore, we can write

$$
\begin{aligned}
\frac{\mathrm{d} w}{\mathrm{~d} z} & =k\left[1-\frac{1+2 \sqrt{2}}{z^{2}-2}\right]^{\frac{1}{4}}=\frac{\partial v}{\partial y}+\mathrm{i} \frac{\partial v}{\partial x} \\
h_{x} & =\sqrt{\left(\frac{\partial u}{\partial x}\right)^{2}+\left(\frac{\partial v}{\partial x}\right)^{2}}=\sqrt{\left(\frac{\partial v}{\partial x}\right)^{2}+\left(\frac{\partial v}{\partial y}\right)^{2}} \\
h_{y} & =\sqrt{\left(\frac{\partial u}{\partial y}\right)^{2}+\left(\frac{\partial v}{\partial y}\right)^{2}}=\sqrt{\left(\frac{\partial v}{\partial x}\right)^{2}+\left(\frac{\partial v}{\partial y}\right)^{2}},
\end{aligned}
$$

i.e.,

$h_{x}=h_{y}$,

and

$$
\begin{gathered}
J(x, y)=\left|\frac{\partial(u, v)}{\partial(x, y)}\right|=\left|\begin{array}{ll}
\frac{\partial u}{\partial x} & \frac{\partial u}{\partial y} \\
\frac{\partial v}{\partial x} & \frac{\partial v}{\partial y}
\end{array}\right| \\
=\frac{\partial u}{\partial x} \cdot \frac{\partial v}{\partial y}-\frac{\partial u}{\partial y} \cdot \frac{\partial v}{\partial x} \\
=\left(\frac{\partial v}{\partial x}\right)^{2}+\left(\frac{\partial v}{\partial y}\right)^{2}=\left|\frac{\mathrm{d} w}{\mathrm{~d} z}\right|^{2} .
\end{gathered}
$$

Therefore, we can write

$$
J(x, y)=h_{x}^{2}=h_{y}^{2}=\left|\frac{\mathrm{d} w}{\mathrm{~d} z}\right|^{2}=|k|^{2}\left|1-\frac{1+2 \sqrt{2}}{z^{2}-2}\right|^{\frac{1}{2}} .
$$

\section{Appendix 4: Fourier orthogonal basis function}

In the space of $L^{2}([a, a+L], m ; C)$, let us set $d_{n}(x)=L^{-1 / 2} \exp \left\{\frac{2 \pi i n(x-a)}{L}\right\}, n \in Z$, which forms a set of orthogonal canonical bases. For any continuous measureable function, we can write

$f(x) \sim \sum_{n=-\infty}^{\infty} C_{n} d_{n}(x), C_{n}=\int_{a}^{a+L} f(x) \overline{d_{n}(x)} d x, \quad n \in Z$

where $d_{n}(x)$ satisfies the following equation:

$d_{n}^{\prime \prime}(x)+\frac{4 \pi^{2} n}{L^{2}} d_{n}(x)=0$.

Then, for $\eta \in\left[\delta_{0}, 1\right]$, we obtain $a=\delta_{0}, L=1-\delta_{0}, \varphi_{k}(\eta)=$ $\frac{1}{\left(1-\delta_{0}\right)^{1 / 2}} \exp \left\{\frac{2 \pi i k\left(\eta-\delta_{0}\right)}{1-\delta_{0}}\right\}$, and $\beta_{k}=\frac{4 \pi^{2} k}{\left(1-\delta_{0}\right)^{2}}$.If $\xi \in[-L, L]$, then $\quad a=-L, L=2 L, \psi_{l}(\xi)=\frac{1}{(2 L)^{1 / 2}} \exp \left\{\frac{2 \pi i l(\xi+L)}{2 L}\right\}$, and $\alpha_{l}=\frac{4 \pi^{2} l}{4 L^{2}}$

\section{References}

1. Caldwell J, Kwan YY (2003) On the perturbation method for the Stefan problem with time-dependent boundary conditions. Int $\mathrm{J}$ Heat Mass Transf 46:1497-1501

2. Myers TG, Mitchell SL (2011) Application of the combined integral method to Stefan problems. Appl Math Model 35:4282-4294

3. Goto T, Suzuki M (1996) Analysis of transient heat conduction with phase changes by a boundary integral equation method. Nucl Eng Des 162:317-324

4. Căruntu B, Bota C (2012) Approximate polynomial solutions for nonlinear heat transfer problems using the squared remainder minimization method. Int Commun Heat Mass Transf 39:1336-1341

5. Yigit $\mathrm{F}$ (2008) Approximate analytical and numerical solutions for a two-dimensional Stefan problem. Appl Math Comput 202:857-869

6. Aziz A, Benzies JY (1976) Application of perturbation techniques to heat-transfer problems with variable thermal properties. Int J Heat Mass Transf 19(3):271-276

7. Villatoro FR, Perez J, Santander JLG, Borovsky MA, Ratis Y, Izzheurov EA (2011) Perturbation analysis of heat transfer in porous media with small thermal conductivity. J Math Anal Appl 374:57-70

8. Sadat H, Prax C (2011) Improved low-order models for heat conduction problems. Int J Heat Mass Transf 54:3789-3795

9. Bau HH (1984) Thermal convection in a horizontal, eccentric annulus containing a saturated porous medium — an extended perturbation expansion. Int J Heat Mass Transf 27(12):2277-2287

10. Joshi Y, Gebhart B (1984) Vertical natural convection flows in porous media: calculations of improved accuracy. Int J Heat Mass Transf 27(1):67-75

11. Jiji LM, Weinbaum S (1978) Perturbation solutions for melting or freezing in annular regions initially not at the fusion temperature. Int J Heat Mass Transf 21(5):581-692 
12. Gupta SC (1987) Analytical and numerical solutions of radially symmetric inward solidification problems in spherical geometry. Int J Heat Mass Transf 30(12):2611-2616

13. Chung M, Jung P-S, Rangel RH (1999) Semi-analytical solution for heat transfer from a buried pipe with convection on the exposed surface. Int J Heat Mass Transf 42:3771-3786

14. Schinzinger R, Laura PAA (2003) Conformal mapping: methods and applications, 1st edn. Dover Publications, INC., Mineola

15. Lai YM, Wang QS, Niu FJ et al (2004) Three-dimensional nonlinear analysis for temperature characteristic of ventilated embankment in permafrost regions. Cold Reg Sci Technol 38(2):165-184

16. Crank J (1981) How to deal with moving boundaries in thermal problems. In: Lewis RW, Morgan K, Zienckiewicz OC (eds) Numerical methods in heat transfer. Wiley, Chichester, pp 177-200

17. Dalhuijsen AJ, Segal A (1986) Comparison of finite element techniques for solidification problems. Int J Numer Methods Eng 23:1807-1829

18. Floryan JM, Zemach C (1993) Schwarz-Christoffel methods for conformal mapping of regions with a periodic boundary. J Comput Appl Math 46:77-102

19. Carslaw HS, Jaeger JC (1959) Conduction of heat in solids. Clarendon Press, Oxford
20. Chen-to T (1986) Unified definition of divergence, curl, and gradient. Appl Math Mech 7(1):1-5 (in Chinese)

21. Hill J (1987) One-dimensional Stefan problem: an introduction. Longman Scientific and Technical, Essex

22. Ozisik MN (1980) Heat conduction. Wiley, New York

23. Nansheng L, Hongsheng L, Dewen D (1997) Quasi-static temperature field and thermal engineering formula of buried-shallowly oil pipeline under severe climate condition. J Glaciol Geocryol 19:65-72 (in Chinese)

24. Aziz A, Na TY (1984) Perturbation methods in heat transfer. Hemisphere, Washington

25. Nayfeh AH (1981) Introduction to perturbation technique. Wiley, New York

26. Reddy JN (1986) Applied functional analysis and variational methods in engineering. McGraw-Hill Book Company, New York

27. Trefethen LN (2000) Spectral methods in MATLAB. SIAM, Philadelphia

28. Boyd JP (2000) Chebyshev and Fourier spectral methods, 2nd edn. DOVER Publications Inc., New York 